\title{
Novel Properties of Atomically Arranged Perovskites
}

\author{
B. Dabrowski, S. Kolesnik, O. Chmaissem, L. Suescun* \\ AND J. MAIS
}

Department of Physics, Northern Illinois University, DeKalb, IL 60115, USA

Perovskites $\mathrm{AMO}_{3-\delta}$ attain unique electronic, magnetic, ferroelectric, thermoelectric, mixed-conducting, and other functional properties through selection of the A- and M-site ions, their fractions, ionic sizes and valences, spin states, and orbital orderings, as well as the oxygen content and vacancy ordering. We illustrate here our systematic exploration of the effects of composition, temperature, pressure, and oxygen content on the thermodynamic stability and magnetic properties for $\mathrm{La}_{1-x-y} \mathrm{Sr}_{x} \mathrm{Ba}_{y} \mathrm{MnO}_{3}$ manganites. Parameters controlling magnetic transitions are identified through examples of single-valent compounds of $\mathrm{RMnO}_{3}$ (the $\mathrm{Mn}-\mathrm{O}-\mathrm{Mn}$ bond angles that can be equivalently described in terms of the tolerance factor) and $\mathrm{Sr}_{1-x} \mathrm{Ca}_{x} \mathrm{MnO}_{3}$ (the tolerance factor and disorder described in terms of the variance of A-site ion sizes), and the mixed-valent $\mathrm{La}_{0.5} \mathrm{Sr}_{0.5-y} \mathrm{Ba}_{y} \mathrm{MnO}_{3}$ (the tolerance factor, variance of sizes, and the local strains described in terms of the elongated Mn-O bonds). By using an example of kinetically stable, atomically-ordered layered-perovskites we show that improved useful properties, such as the increase in the Curie temperature $T_{\mathrm{C}}$ and enhancement of colossal magnetoresistive effect at room temperature, can be achieved through decreases in structural distortions such as variance of sizes and local strains.

PACS numbers: 75.47.Lx, 75.47.Gk, 61.12.Ld

\section{Introduction}

Owing to unusual chemical versatility and structural intricacy perovskite compounds $\mathrm{AMO}_{3-\delta}$ can be designed to attain unique electronic, magnetic, ferroelectric, and other properties through selection of the A- and M-site ions, their fractions, ionic sizes and valences, spin states, and orbital orderings, as well as the oxygen content and vacancy ordering. The best-known examples are spectacular high temperature superconducting cuprates (for example Y-123, $\left.\mathrm{Y}_{1 / 3} \mathrm{Ba}_{2 / 3} \mathrm{CuO}_{7 / 3-\delta}\right)$ [1], colossal magnetoresistive manganites [2], and recently

*Permanent address: Cryssmat-Lab/Cátedra de Física/DETEMA, Facultad de Química, Universidad de la República, P.O.Box 1157, Montevideo, Uruguay. 
discovered remarkable cobaltites with spin-dependent thermoelectric power and conductivity [3]. These substances are characterized by strong electron-spinphonon interactions that lead to correlation between structural order (for example, oxygen vacancies and layer order of $\mathrm{A} / \mathrm{A}^{\prime}$ and $\mathrm{M} / \mathrm{M}^{\prime}$ ions) and electronic order (for example, superconducting, charge, magnetic, orbital, and spin). Thus, complex oxides are now emerging as remarkably adaptable materials that will play an important role in the next generation of electronic industry and in energy generation, conversion, transmission, and usage. Design of new compounds with tailored characteristics coupled to in-depth investigation of their fundamental properties and correlation of the properties to various orderings and interaction strengths are useful to demonstrate the potential of these materials for applications.

We have been systematically developing an understanding of the relationship between properties of perovskite compounds and their chemical composition, structure, and synthesis conditions to enable the preparation of improved materials and discovery of new compounds. In previous paper we have reviewed strategies used for designing and preparing new perovskite manganites with enhanced magnetoresistive properties and increased magnetic transition temperatures [4]. This effort requires a twofold approach: understanding of the dependence of properties on chemical and structural factors and development of ability for synthesis of desired compounds. In this paper we illustrate our systematic exploration of the effects of composition, temperature, and oxygen content on thermodynamic stability and physical properties of the $\mathrm{La}_{1-x-y} \mathrm{Sr}_{x} \mathrm{Ba}_{y} \mathrm{MnO}_{3}$ manganites. Our description will use internal structural parameters such as the $\mathrm{Mn}-\mathrm{O}-\mathrm{Mn}$ bond angles and the $[\mathrm{A}-\mathrm{O}]$ and $[\mathrm{Mn}-\mathrm{O}]$ bond lengths, which are obtained with high accuracy from highresolution neutron diffraction measurements. These structural measurements are combined with detailed magnetic and resistive measurements that are performed over wide temperature ranges to provide accurate composition-structure-property phase diagrams, which can be used to discover better materials. First, we will identify increasingly more complex structural parameters, which are controlling magnetic transitions $T_{\mathrm{M}}$ in randomly substituted $(x, y)$ perovskites on the A-site. The simplest parameter is the average bond angle $\theta=\langle\mathrm{Mn}-\mathrm{O}-\mathrm{Mn}\rangle$ (which can be equivalently described in terms of the tolerance factor $t=[\mathrm{A}-\mathrm{O}] / \sqrt{2}[\mathrm{Mn}-\mathrm{O}]$ ) for the single-valent $\mathrm{RMn}^{3}+\mathrm{O}_{3}$ compounds with only one kind of $\mathrm{R}$ ion located on the A-site [5]. Two parameters, $t(x, y)$ and the variance of the A-site ion sizes $\sigma(x)=\sqrt{\Sigma_{i}\left\{x_{i}\left[\mathrm{~A}_{i}-\mathrm{O}\right]^{2}-[\mathrm{A}-\mathrm{O}]^{2}\right\}}$ (describing the disorder of bond angles $\left.\mathrm{Mn}-\mathrm{O}-\mathrm{Mn},[\mathrm{A}-\mathrm{O}]=\sum_{i} x_{i}\left[\mathrm{~A}_{i}-\mathrm{O}\right]\right)$ were recently shown [6] to provide more precise description for $\mathrm{Sr}_{1-x} \mathrm{Ca}_{x} \mathrm{Mn}^{4+} \mathrm{O}_{3}$ compounds with two (or more, for example, $\mathrm{La}_{1-x-y} \mathrm{Sr}_{x} \mathrm{Ba}_{y} \mathrm{MnO}_{3}$ ) different cations located on the A-site [7]. According to such a two-parameter model [8], magnetic materials with cubic symmetries, $t(x, y)=1$, and small $\sigma(x)$ should have maximal $T_{\mathrm{M}}$ 's.

We will show that three parameters $t(x, y), \sigma(x)$, and local strains (which can be described in terms of the elongated [Mn-O] bonds) [9] are necessary for correct 
description of suppressed $T_{\mathrm{C}}$ for nearly cubic or cubic $\mathrm{La}_{0.5} \mathrm{Sr}_{0.5-y} \mathrm{Ba}_{y} \mathrm{Mn}^{3.5+} \mathrm{O}_{3}$. The recently identified parameter of the local strains is responsible for the universally observed suppression of $T_{\mathrm{C}}$ for slightly distorted and cubic symmetries contrary to the two-parameter model. We will show that through decreases in the structural distortions, i.e., the variance of sizes and local strains, improved useful properties such as an increase in $T_{\mathrm{C}}$ and enhancement of the colossal magnetoresistive effect at room temperature can be achieved for kinetically stable and atomically ordered layered-perovskites $\mathrm{RBaMn}_{2} \mathrm{O}_{6}[10-12]$.

\section{Kinetically stable $\mathrm{La}_{1-x-y} \mathrm{Sr}_{x} \mathrm{Ba}_{y} \mathrm{MnO}_{3}$ perovskites}

We have recently shown that the geometrical concept of tolerance factor can be reliably used to describe the structural stability of the perovskite phase when the effects of varying synthesis temperature $(T)$ and oxygen content $(3-\delta)$ are correctly included in definition of tolerance factor in addition to the usual dependence on composition ( $x$ or $y$ ) [7]. By using special synthesis techniques knowledge of $t(x, y, T, \delta)$ allows to extend chemical composition ranges far beyond those previously achieved for the $\mathrm{La}_{1-x-y} \mathrm{Sr}_{x} \mathrm{Ba}_{y} \mathrm{MnO}_{3}$ manganites. Fig-

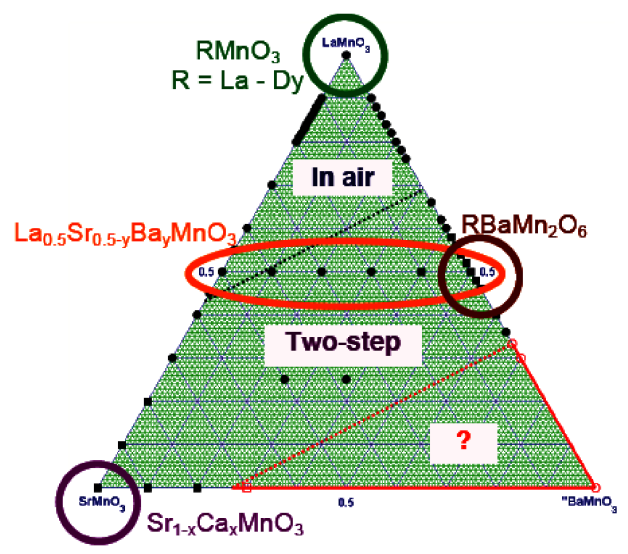

Fig. 1. Composition ranges for perovskites $\mathrm{La}_{1-x-y} \mathrm{Sr}_{x} \mathrm{Ba}_{y} \mathrm{MnO}_{3}$ synthesized: "In air" — previously known, "Two step" — obtained by a two-step synthesis method, "?" not achieved to date. The observed average simple cubic and non-cubic crystal structures are denoted by filled squares and filled circles, respectively. Compounds discussed in the text are pointed out.

ure 1 shows the compositional ranges achieved in air and by the two-step synthesis method at reduced oxygen pressure. Fully oxidized compounds are subsequently obtained by anneal in oxygen at low temperatures where the oxygen diffusion is fast enough to fill oxygen vacancies but cationic diffusion is negligible, i.e., the perovskite phase is kinetically stable. In the figure there are also denoted compositional ranges which will be discussed in the following sections: 
$\mathrm{RMnO}_{3}, \mathrm{Sr}_{1-x} \mathrm{Ca}_{x} \mathrm{MnO}_{3}, \mathrm{La}_{0.5} \mathrm{Sr}_{0.5-y} \mathrm{Ba}_{y} \mathrm{Mn}^{3.5+} \mathrm{O}_{3}$, and $\mathrm{RBaMn}_{2} \mathrm{O}_{6}$. While the randomly-substituted $\mathrm{Sr}_{1-x} \mathrm{Ca}_{x} \mathrm{MnO}_{3}$ and $\mathrm{La}_{0.5} \mathrm{Sr}_{0.5-y} \mathrm{Ba}_{y} \mathrm{MnO}_{3}$ compounds could be relatively easily obtained in Ar, the synthesis of a well layered-ordered $\mathrm{RBaMn}_{2} \mathrm{O}_{6}$ compounds required accurate control of the oxygen content near $\mathrm{O}_{5}$ at high temperatures by using in situ thermogravimetric analysis (TGA) apparatus with $\mathrm{H}_{2} /$ Ar flow $[4,10,13]$.

\section{Dependence of the exchange interactions on structural parameters}

Chemical compositions discussed here span a range of the Mn oxidation states from $\mathrm{Mn}^{3+}$ to $\mathrm{Mn}^{4+}$. It is well known from the Hund and crystal-field splitting rules that these oxidation states correspond to the electronic ground states from $t^{3} e^{1}$ to $t^{3} e^{0}$. It has been previously shown that the Jahn-Teller distortion and the Goodenough-Kanamori rules of exchange interactions lead to the antiferromagnetic and ferromagnetic indirect interactions which are dependent on the bond lengths $[\mathrm{Mn}-\mathrm{O}]$ and bond angles $\theta$ through the phenomenological relationship $J \sim W^{2} \sim\left\{\cos \omega /[\mathrm{Mn}-\mathrm{O}]^{3.5}\right\}^{2}$, where $W$ is the band width and $\omega=(180-\theta) / 2[14]$. When the bond lengths $[\mathrm{Mn}-\mathrm{O}]$ are constant for various substitutions on the A-site but the $\mathrm{Mn}-\mathrm{O}-\mathrm{Mn}$ bonds $\theta$ are not straight and altering, the interactions can be described simply as a superposition of 180 and $90 \mathrm{deg}$ bonds leading to the formula for magnetic transition temperatures $T_{\mathrm{M}} \sim J \approx T_{\mathrm{M} \max }-T_{1}\left(1-\left\langle\cos ^{2} \theta\right\rangle\right)[10,11]$. Such a relationship has been observed for the Néel temperatures $T_{\mathrm{N}}$ for single valent $\mathrm{RMO}_{3}$ systems with $\mathrm{M}=\mathrm{Fe}^{3+}\left(t^{3} e^{2}\right), \mathrm{Cr}^{3+}\left(t^{3}\right)$, and $\mathrm{Ni}^{3+}\left(t^{6} e^{1}\right)$ when there is only one kind of rare earth on the A-site [15].
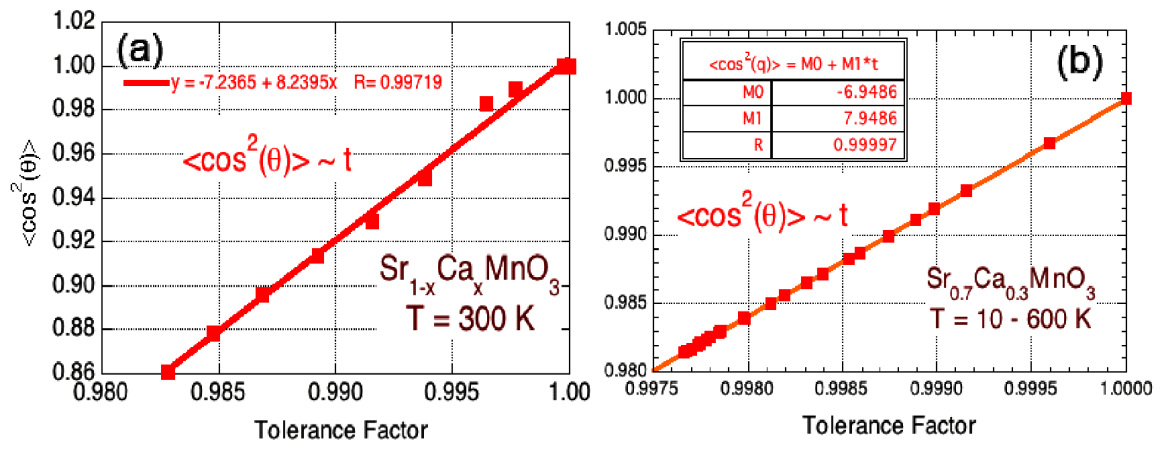

Fig. 2. Dependence of $\left\langle\cos ^{2}(\theta)\right\rangle$ on tolerance factor for $\mathrm{Sr}_{1-x} \mathrm{Ca}_{x} \mathrm{MnO}_{3}$ (a) at room temperature and (b) for temperatures $10-600 \mathrm{~K}$.

Figure 2a shows linear relationship between $\left\langle\cos ^{2} \theta\right\rangle$ and tolerance factor $t(x)$ measured at $300 \mathrm{~K}$ as a function of composition for more complex single valent system $\mathrm{Sr}_{1-x} \mathrm{Ca}_{x} \mathrm{MnO}_{3}$. Since we have observed that a similar linear relationship is universally observed for manganites also as a function of temperature (see 
Fig. 2b), a simpler relation could be derived for dependence of magnetic transition temperatures on tolerance factor $T_{\mathrm{M}}=T_{0}-\alpha[1-t(x)]$. The use of tolerance factor rather than the bond angles $\theta$ is of great advantage for description of magnetic and resistive behavior since once measured, bond lengths $[\mathrm{A}-\mathrm{O}]$ and $[\mathrm{Mn}-\mathrm{O}]$ are tabulated, and there is no need to measure $\theta$ for any combination of the A-ions to calculate $t(x)$ and $\sigma(x)$, and to predict $T_{\mathrm{M}}[7]$.

\section{Magnetic behavior for single-valent systems}

We have recently shown by means of the direct magnetic and structural measurements for single valent perovskites that the linear relationship between the $T_{\mathrm{N}}$ and $\left\langle\cos ^{2} \theta\right\rangle$ holds very well for the $\mathrm{RMnO}_{3}$ system with $\mathrm{Mn}^{3+}$ while for the solid solution system $\mathrm{Sr}_{1-x} \mathrm{Ca}_{x} \mathrm{MnO}_{3}$ with $\mathrm{Mn}^{4+}$ the structural disorder on the A-site $\sigma(x)$ introduces clear deviation from this relationship [7, 16]. Figure 3 shows a summary of $T_{\mathrm{N}}$ dependence on $\left\langle\cos ^{2} \theta\right\rangle$ and $\sigma(x)$ for both systems.

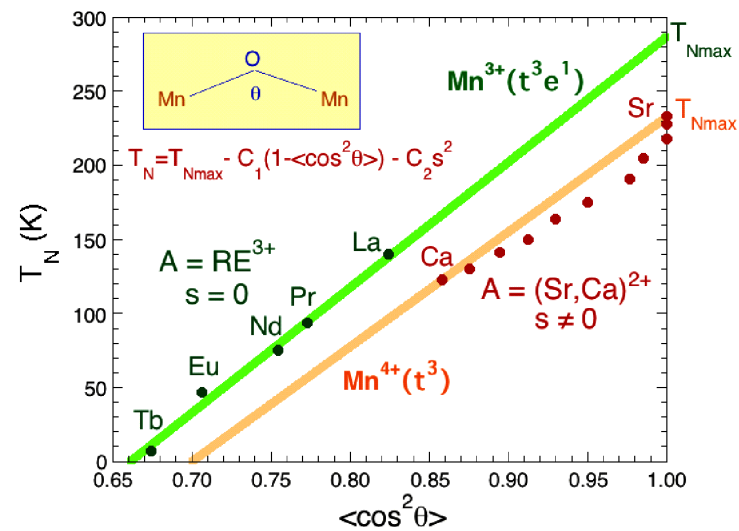

Fig. 3. Summary of $T_{\mathrm{N}}$ dependence on $\left\langle\cos ^{2} \theta\right\rangle$ and $\sigma(x)$ for $\mathrm{RMnO}_{3}$ and $\mathrm{Sr}_{1-x} \mathrm{Ca}_{x} \mathrm{MnO}_{3}$ systems.

In Fig. 4a we show that the use of the simplified formulae $J \sim$ $\left\{\cos \omega /[\mathrm{Mn}-\mathrm{O}]^{3.5}\right\}^{2}$ with $[\mathrm{Mn}-\mathrm{O}]=$ constant is justified for the $\mathrm{RMnO}_{3}$ system. When various rare earths are substituted on the A-site the bond lengths $[\mathrm{A}-\mathrm{O}]$ scale with the bond angles $\theta$, and they increase with the ionic size of $\mathrm{R}$. The bond length $\left[\mathrm{Mn}^{3+}-\mathrm{O}\right]$ remains, however, constant, as expected for the $\mathrm{Mn}^{3+}$ ion with a fixed oxidation state for all compounds. We note that the ratio of $[\mathrm{A}-\mathrm{O}]$ to $\sqrt{2}[\mathrm{~B}-\mathrm{O}]$ (i.e., the tolerance factor) is considerably smaller than 1 for all $\mathrm{R}$, and as such, all $\mathrm{RMnO}_{3}$ compounds exhibit a highly distorted orthorhombic structure with localized Jahn-Teller distortions. In Fig. 4b we show that also for the $\mathrm{Sr}_{1-x} \mathrm{Ca}_{x} \mathrm{MnO}_{3}$ system the bond length $\left[\mathrm{Mn}^{4+}-\mathrm{O}\right]$ remains constant as long as the structure is non-cubic, i.e., for orthorhombic (highly distorted) and tetragonal (slightly distorted) phases. As the larger $\mathrm{Sr}$ and $\mathrm{Ba}$ are substituted for the cubic 

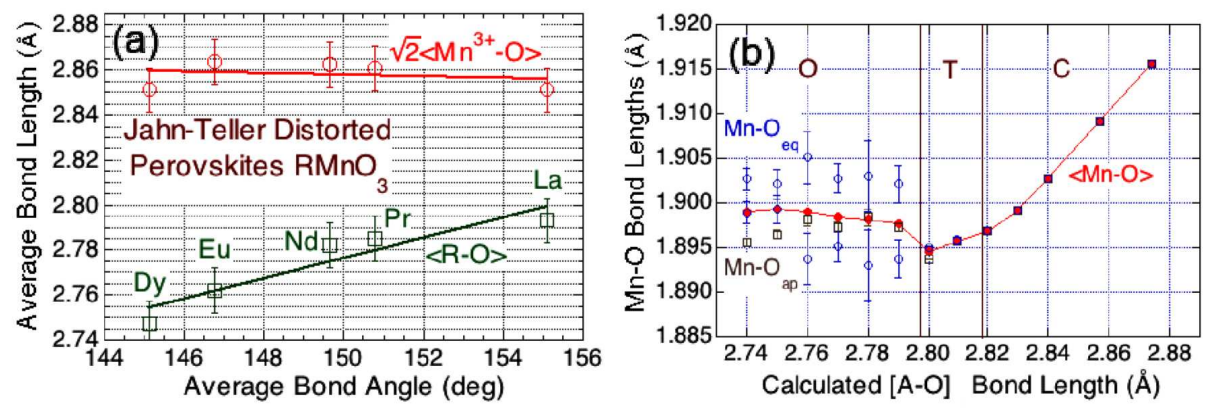

Fig. 4. (a) Dependence of the bond lengths $\left[\mathrm{Mn}^{3+}-\mathrm{O}\right]$ and $[\mathrm{R}-\mathrm{O}]$ on bond angles $\theta$ for $\mathrm{RMnO}_{3}$. (b) Dependence of the bond lengths $\left[\mathrm{Mn}^{4+}-\mathrm{O}\right]$ on tabulated bond length [A-O] (from Ref. [22]) for $\mathrm{Sr}_{1-x} \mathrm{Ca}_{x} \mathrm{MnO}_{3}$.

crystal structure the symmetry constraint (matching of the bond lengths $[\mathrm{A}-\mathrm{O}]$ and $\sqrt{2}[\mathrm{M}-\mathrm{O}])$ puts $\left[\mathrm{Mn}^{4+}-\mathrm{O}\right]$ bond under tension and causes it to expand beyond its equilibrium size of $1.897 \pm 0.002 \AA$. This expansion, together with a considerable increase in the structural disorder $\sigma(x)$ on the A-site is responsible for a rapid decrease in $T_{\mathrm{N}}$ for the $\mathrm{Sr}_{1-x} \mathrm{Ba}_{x} \mathrm{MnO}_{3}$ system $[7,16]$.

\section{Magnetic behavior for mixed-valent system near transition to cubic structure}

Our neutron diffraction studies provided accurate measurements of the bond lengths $\left[\mathrm{Mn}^{3+}-\mathrm{O}\right]=1.980 \pm 0.006$ (for itinerant case), $[\mathrm{La}-\mathrm{O}]=2.769 \pm 0.003$, $[\mathrm{Sr}-\mathrm{O}]=2.691 \pm 0.002$, and $[\mathrm{Ba}-\mathrm{O}]=2.783 \pm 0.005 \AA$ for a wide range of compositions shown in Fig. 1. As $\mathrm{Sr}$ and $\mathrm{Ba}$ are substituted for La the tolerance factor $t(x, y)$ increases and the structures of the $\mathrm{La}_{1-x-y} \mathrm{Sr}_{x} \mathrm{Ba}_{y} \mathrm{MnO}_{3}$ compounds evolve toward less distorted cubic symmetry. Figure 1 shows the compositions for which cubic (filled squares) and non-cubic (filled circles) structures were found. It has been previously observed that samples with a fixed doping level near $x+y=0.3-0.5$ exhibit ferromagnetic properties with the Curie temperature, $T_{\mathrm{C}}$, decreasing as a function of increasing Ba content. Decreasing $T_{\mathrm{C}}$ observed in $\mathrm{La}_{0.6} \mathrm{Sr}_{0.6-x} \mathrm{Ba}_{x} \mathrm{MnO}_{3}[17,18], \mathrm{Pr}_{0.5} \mathrm{Sr}_{0.5-x} \mathrm{Ba}_{x} \mathrm{MnO}_{3}$ [19, 20], and $\mathrm{Nd}_{0.7} \mathrm{Sr}_{0.3-x} \mathrm{Ba}_{x} \mathrm{MnO}_{3}$ [21] was interpreted in terms of increasing ionic size variance $s(x)$ or increasing [Mn-O] bond lengths. Both of these arguments mainly relied on Shannon's tabulated ionic sizes [22] that differ appreciably from the observed real values for manganites. Here we show that the explanation of $T_{\mathrm{C}}$ behavior only in terms of tolerance factor and size variance is not sufficient especially in the regime where structural distortions from the cubic structure are very small.

Figure 5 shows measured bond lengths $\left[\mathrm{Mn}^{3.5+-} \mathrm{O}\right]$ and bond angles $\mathrm{Mn}-\mathrm{O}-\mathrm{Mn}$ as a function of Ba content at room temperature. Compositions $x=$ 0-0.2 and 0.3-0.5 are tetragonal (slightly distorted) and cubic, respectively. Bond 


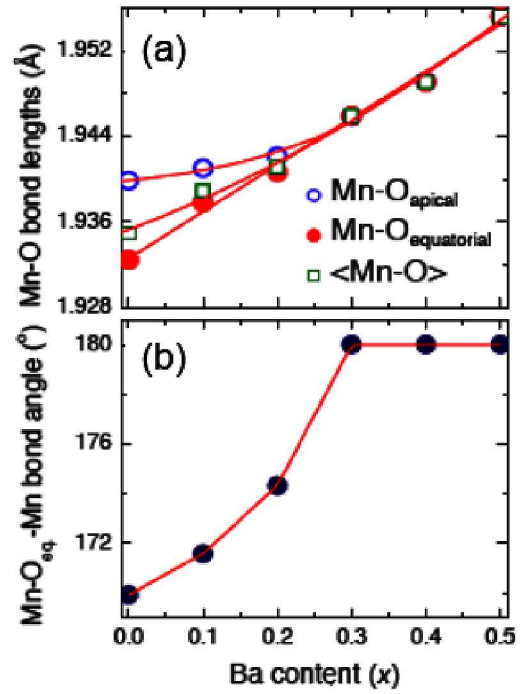

Fig. 5. Bond lengths $\left[\mathrm{Mn}^{3.5+}-\mathrm{O}\right]$ (a) and bond angles $[\mathrm{Mn}-\mathrm{O}-\mathrm{Mn}]$ (b) as a function of Ba content for $\mathrm{La}_{1-x-y}^{3+} \mathrm{Sr}_{x}^{2+} \mathrm{Ba}_{y}^{2+} \mathrm{Mn}^{3+x+y} \mathrm{O}_{3}$ compounds at room temperature.

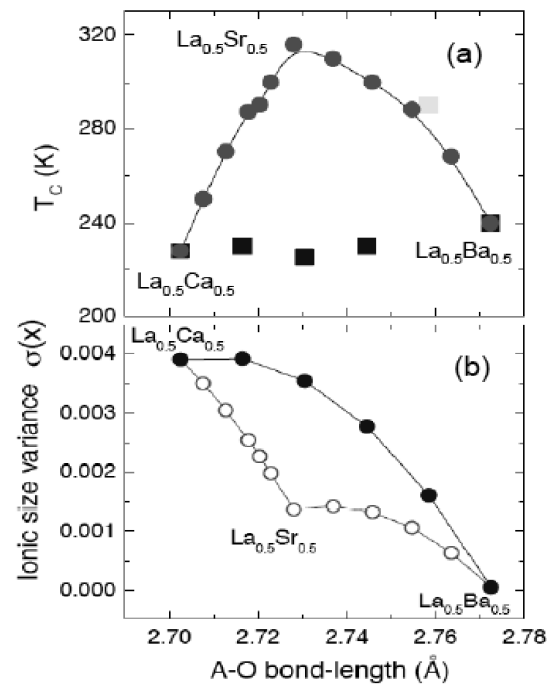

Fig. 6. (a) Curie temperature $T_{\mathrm{C}}$ and (b) variance of sizes $\sigma(x)$ as a function of the bond length $[\mathrm{A}-\mathrm{O}]$ for $\left(\mathrm{La}_{0.5} \mathrm{~A}_{0.5}\right) \mathrm{MnO}_{3}$ compounds $(\mathrm{A}=\mathrm{Ca}, \mathrm{Sr}, \mathrm{Ba})$.

lengths $\left[\mathrm{Mn}^{3.5+}-\mathrm{O}\right]$ exhibit a small increase already in non-cubic phase $(t(x)<1)$ and a more rapid increase imposed by requirement of matching bond lengths $[\mathrm{A}-\mathrm{O}]$ and $\sqrt{2}\left[\mathrm{Mn}^{3.5+}-\mathrm{O}\right]$ in cubic phase $(t(x)=1)$. Figure 6 shows $T_{\mathrm{C}}$ and variance of sizes $\sigma(x)$ as a function of the bond length [A-O] for several $\left(\mathrm{La}_{0.5} \mathrm{~A}_{0.5}\right) \mathrm{MnO}_{3}$ compounds $(\mathrm{A}=\mathrm{Ca}, \mathrm{Sr}, \mathrm{Ba})$. We observe that 
$T_{\mathrm{C}}$ decreases for both tetragonal and cubic phases despite increasing tolerance factor and decreasing variance of sizes. Thus, the explanation of the observed $T_{\mathrm{C}}$ reduction in terms of increasing ionic size variance cannot be employed when the accurately measured bond lengths are used. Clearly additional mechanism is required when the crystal structure is cubic or nearly cubic. Such mechanism can arise from the presence of the $[\mathrm{Mn}-\mathrm{O}]$ bonds that are stretched beyond their equilibrium values. A fraction of such bonds would be present because the equilibrium $\sqrt{2}[\mathrm{Mn}-\mathrm{O}]$ bonds are smaller than the longest $[\mathrm{A}-\mathrm{O}]$ bonds (i.e., the $[\mathrm{Ba}-\mathrm{O}]$ bonds) even if the average structure is non-cubic, i.e., when the tolerance factor is still smaller than 1, i.e., the average bond angle $\mathrm{Mn}-\mathrm{O}-\mathrm{Mn} \neq 180 \mathrm{deg}$. As the formula for the band width $W$ shows, these bonds would cause a very strong and non-linear decrease in $T_{\mathrm{C}}$ that is not modeled by the variance of sizes $\sigma(x)$. The presence of stretched out [Mn-O] bonds caused by the substitutions of larger $\mathrm{Ba}$ ions for $\mathrm{Sr}$ in the lattice as the materials become cubic, indicates the development of considerable internal strains that cause lowering of $T_{\mathrm{C}}$.

\section{Enhancement of magnetic properties in layered-perovskites}

As we have seen above, the rare earth and alkaline earth ions of different sizes and charges introduced into the A-sites of the perovskite structure distort bond lengths $[\mathrm{Mn}-\mathrm{O}]$ and bond angles $\mathrm{Mn}-\mathrm{O}-\mathrm{Mn}$, causing local disorder that is cooperatively propagated through the lattice via the shared oxygen in adjacent $\mathrm{MnO}_{6}$ octahedra. The half-doped manganites $\mathrm{RBaMn}_{2} \mathrm{O}_{6}$ provide rich opportunity for investigations of the interplay between distortions and various types of magnetic and charge ordering. Half-doped manganites can be prepared in two phases; as normal perovskites with randomly mixed A-site ions and the A-site ordered perovskite structures in which the layered $c$-axis is composed of alternating RO and $\mathrm{BaO}$ sheets separated by sheets of $\mathrm{MnO}_{2}$ [23]. Reducing chemical disorder through A-site ordering produces "cleaner" materials that exhibit enhanced magnetic prop-

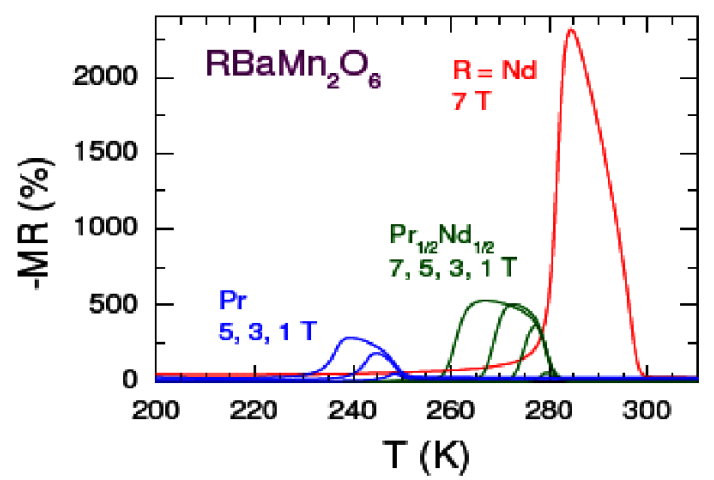

Fig. 7. Magnetoresistive effect for layer ordered perovskites $\mathrm{RBaM}_{2} \mathrm{O}_{6}$. 


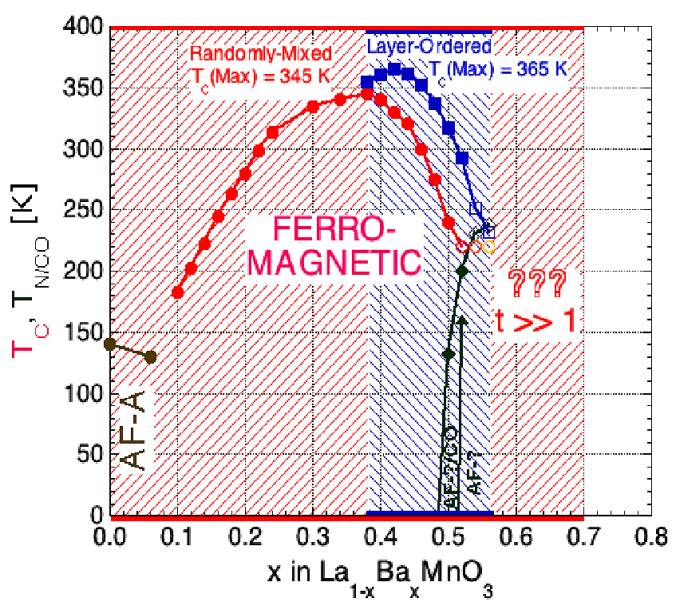

Fig. 8. Compositions and magnetic transition temperatures achieved for the normal and layer-ordered perovskites of $\mathrm{La}_{1-x} \mathrm{Ba}_{x} \mathrm{MnO}_{3}$.

erties [10]. Complex metal-insulator transitions and charge, orbital, and magnetic orderings have been observed over a wide temperature range that reaches $550 \mathrm{~K}$ for half-doped $\mathrm{RBaMn}_{2}^{3.5+} \mathrm{O}_{6}$ materials [24]. We have studied these compounds in an attempt to obtain enhanced colossal magnetoresistance (CMR) effects by exploiting the proximity of the ferromagnetic (FM) and antiferromagnetic (AF) transitions near $300 \mathrm{~K}$ [10-12]. Large CMR effects were indeed observed as shown in Fig. 7, but, unfortunately, they have turned out to be ineffectual because of the first order nature of the encountered phase transitions. We were, however, able to stabilize the layered perovskite of $\mathrm{La}_{1-x} \mathrm{Ba}_{1+x} \mathrm{Mn}_{2} \mathrm{O}_{6}$ over the range of compositions $-0.04<x<0.10$ and increase the maximum $T_{\mathrm{C}}$ from 345 to $365 \mathrm{~K}$ by suppressing local structural and charge disorder of the randomly mixed phase. Figure 8 shows achieved layered perovskites and randomly mixed compositions and their magnetic transition temperatures for the $\mathrm{La}_{1-x} \mathrm{Ba}_{x} \mathrm{MnO}_{3}$ system. To date we were not able to stabilize similar layered perovskite $\mathrm{La}_{1-x} \mathrm{Sr}_{1+x} \mathrm{Mn}_{2} \mathrm{O}_{6}$ compounds for which $T_{\mathrm{C}}$ of $400 \mathrm{~K}$ is expected.

\section{Conclusions}

Once parameters controlling physical properties of $\mathrm{AMO}_{3}$ perovskites are identified, they can be controlled through selection of the A- and M-site ions, their fractions, ionic sizes and valences, as well as the oxygen content and vacancy ordering. We have illustrated here our systematic exploration of the effects of composition, temperature, and oxygen pressure for achieving new $\mathrm{La}_{1-x-y} \mathrm{Sr}_{x} \mathrm{Ba}_{y} \mathrm{MnO}_{3}$ manganites with enhanced magnetoresistive properties [12]. Parameters control$\operatorname{ling} T_{\mathrm{M}}$ were identified using examples of single-valent compounds of $\mathrm{RMnO}_{3}$ (the $\mathrm{Mn}-\mathrm{O}-\mathrm{Mn}$ bond angles or equivalently the tolerance factor) and $\mathrm{Sr}_{1-x} \mathrm{Ca}_{x} \mathrm{MnO}_{3}$ (the tolerance factor and the variance of A-site ion sizes), and the mixed- 
-valent $\mathrm{La}_{0.5} \mathrm{Sr}_{0.5-y} \mathrm{Ba}_{y} \mathrm{MnO}_{3}$ (the tolerance factor, variance of sizes, and the local strains). By using an example of kinetically stable, atomically-ordered layered-perovskites we have shown that improved useful properties, such as the increase in $T_{\mathrm{C}}$ and enhancement of CMR effect at room temperature, can be achieved through decreases in structural distortions, variance of sizes, and local strains that are typically suppressing magnetic properties.

\section{Acknowledgments}

This work was supported by the NSF grant No. DMR-0302617 and the U.S. Department of Education.

\section{References}

[1] J.G. Bednorz, K.A. Muller, Z. Phys. B 64, 189 (1986).

[2] R.M. Kusters, J. Singelton, D.A. Keen, R. McGreevy, W. Hayes, Physica B 155, 362 (1989).

[3] I.O. Troyanchuk, N.V. Kasper, D.D. Khalyavin, H. Szymczak, R. Szymczak, M. Baran, Phys. Rev. Lett. 80, 3380 (1998).

[4] B. Dabrowski, O. Chmaissem, J. Mais, S. Kolesnik, Acta Phys. Pol. A 105, 45 (2004).

[5] B. Dabrowski, S. Kolesnik, A. Baszczuk, O. Chmaissem, T. Maxwell, J. Mais, J. Solid State Chem. 178, 629 (2005).

[6] J.P. Attfield, A.L. Kharlanov, J.A. McAllister, Nature (London) 394, 157 (1998).

[7] B. Dabrowski, O. Chmaissem, M. Mais, S. Kolesnik, J.D. Jorgensen, S. Short, J. Solid State Chem. 170, 154 (2003).

[8] L.M. Rodriguez-Martinez, J.P. Attfield, Phys. Rev. B 54, R15622 (1996).

[9] O. Chmaissem, B. Dabrowski, S. Kolesnik, J. Mais, T. Maxwell, L. Suescun, J.D. Jorgensen, Phys. Rev. B 74, 144415 (2006).

[10] B. Dabrowski, O. Chmaissem, M. Mais, S. Kolesnik, J.D. Jorgensen, S. Short, Mater. Res. Soc. Symp. Proc. 718, 169 (2002).

[11] B. Dabrowski, O. Chmaissem, S. Kolesnik, J. Mais, J.D. Jorgensen, Physica C 387, 266 (2003).

[12] H. Churchill, Y. Ren, A.N. Styka, B. Dabrowski, J. Mais, S. Kolesnik, O. Chmaissem, D.E. Brown, unpublished results.

[13] O. Chmaissem, B. Dabrowski, S. Kolesnik, J. Mais, J.D. Jorgensen, S. Short, C.E. Botez, P.W. Stephens, Phys. Rev. B 72, 104426 (2005).

[14] W.A. Harrison, The Electronic Structure and Properties of Solids, Freeman, San Francisco 1980.

[15] J.B. Goodenough, Rep. Prog. Phys. 67, 1915 (2004).

[16] O. Chmaissem, B. Dabrowski, S. Kolesnik, J. Mais, D.E. Brown, R. Kruk, P. Prior, B. Pyles, J.D. Jorgensen, Phys. Rev. B 64, 134412 (2001).

[17] N. Abdelmoula, E. Dhahri, K. Guidara, J.C. Joubert, Phase Transit. 69, 215 (1999). 
[18] C.H. Shen, C.C. Chen, R.S. Liu, R. Gundakaram, S.F. Hu, J.M. Chen, J. Solid State Chem. 156, 117 (2001).

[19] F. Damay, C. Martin, A. Maignan, B. Raveau, J. Appl. Phys. 82, 6181 (1997).

[20] C. Autret, C. Martin, M. Hervieu, A. Maignan, B. Raveau, G. Andre, F. Bouree, Z. Jirak, Chem. Mater. 15, 1886 (2003).

[21] E. Suard, F. Fauth, C. Martin, A. Maignan, F. Millange, L. Keller, J. Magn. Magn. Mater. 264, 221 (2003).

[22] R.D. Shannon, Acta Crystallogr., Sect. A: Cryst. Phys., Diffr., Theor. Gen. Crystallogr. 32, 751 (1976).

[23] F. Millange, V. Caignaert, B. Domengès, B. Raveau, E. Suard, Chem. Mater. 10, 1974 (1998).

[24] D. Akahoshi, M. Uchida, Y. Tomioka, T. Arima, Y. Matsui, Y. Tokura, Phys. Rev. Lett. 90, 177203 (2003). 